\title{
Anomalous X-ray Diffraction Studies for Photovoltaic Applications
}

\author{
Grisel Rivera Batista \\ Department of Energy, Science Undergraduate Laboratory Internship Program
}
University of Puerto Rico, Río Piedras Campus
San Juan, Puerto Rico
SLAC National Accelerator Laboratory
Menlo Park, California

August 13, 2010

Prepared in partial fulfillment of the requirement of the Office of Science, Department of Energy's Science Undergraduate Laboratory Internship under the direction of Michael Toney, Sumohan Misra and Joanna Bettinger in the Stanford Synchrotron Radiation Lightsource at SLAC National Accelerator Laboratory.

Participant:

Signature

Research Advisor:

Signature

Research Advisor:

Signature

Research Advisor:

Signature 


\section{TABLE OF CONTENTS}

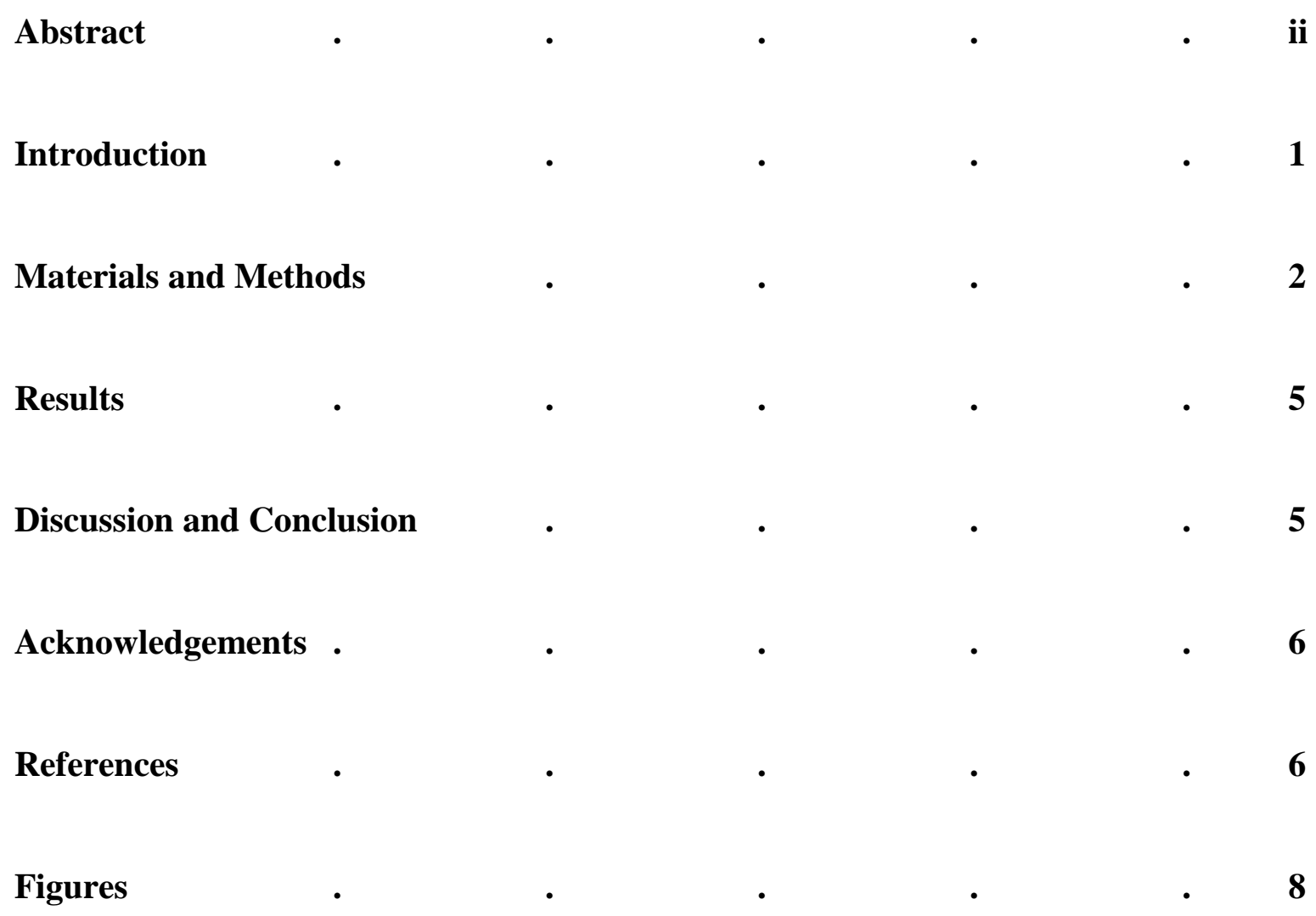




\begin{abstract}
Anomalous X-ray Diffraction Studies for Photovoltaics Applications - Solar Cells, GRISEL RIVERA BATISTA (University of Puerto Rico, Río Piedras Campus, San Juan, PR 00931-1907) MICHAEL TONEY, SUMOHAN MISRA, JOANNA BETTINGER (SLAC National Accelerator Laboratory, Menlo Park, CA 94025)

Anomalous X-ray Diffraction (AXRD) has become a useful technique in characterizing bulk and nanomaterials as it provides specific information about the crystal structure of materials. In this project we present the results of AXRD applied to materials for photovoltaic applications: $\mathrm{ZnO}$ loaded with $\mathrm{Ga}$ and $\mathrm{ZnCo}_{2} \mathrm{O}_{4}$ spinel. The X-ray diffraction data collected for various energies were plotted in Origin software. The peaks were fitted using different functions including Pseudo Voigt, Gaussian, and Lorentzian. This fitting provided the integrated intensity data (peaks area values), which when plotted as a function of X-ray energies determined the material structure. For the first analyzed sample, Ga was not incorporated into the $\mathrm{ZnO}$ crystal structure. For the $\mathrm{ZnCo}_{2} \mathrm{O}_{4}$ spinel Co was found in one or both tetrahedral and octahedral sites.
\end{abstract}




\section{INTRODUCTION}

The use of anomalous X-ray diffraction (AXRD) provides element and site specific information for the crystal structure of a material. This technique lets us correlate the structure to the electronic properties of the materials as it allows us to probe precise locations of cations in the spinel structure. What makes it possible is that in AXRD the diffraction pattern is measured at a number of energies near an X-ray absorption edge of an element of interest. The atomic scattering strength of an element varies near its absorption edge and hence the total intensity of the diffraction peak changes by changing the X-ray energy. Thus AXRD provides element specific structural information. This method can be applied to both crystalline and liquid materials. One of the advantages of AXRD in crystallography experiments is its sensitivity to neighboring elements in the periodic tables. This method is also sensitive to specific crystallographic phases and to a specific site in a phase [1].

The main use of AXRD in this study is for transparent conductors (TCs) analysis. TCs are considered to be important materials because of their efficiency and low risk of environmental pollution. These materials are important to solar cells as a result of their remarkable combination of optical and electrical properties, including high electrical conductivity and high optical transparency in the spectrum of visible light [2][3]. TCs provide a transparent window, which allows sunlight to pass through while also allowing electricity to conduct out of the cell.

Spinel materials have the chemical form $A B_{2} \mathrm{O}_{4}$, and are made of a face-centered cubic (FCC) lattice of oxygen anions and cations in specific interstitial sites (see Figure 1,2) [4] [5]. A normal spinel has all $A$ cations on tetrahedral sites and $B$ cations on octahedral sites. In contrast; an inverse spinel has the $A$ and half of the $B$ cations on octahedral sites and the other half of the $B$ cations on tetrahedral sites; a mixed spinel lies between (see Figure 3). In the spinel structure, 8 
of 64 possible tetrahedral sites and 16 of 32 possible octahedral sites are filled. Normal spinels have particularly high conduction as the linear octahedral chains of $B$ cations likely serve as conduction paths [2].

In this paper we present how the data obtained with AXRD is used to analyze TCs properties as they apply to photovoltaic applications. One of the materials used for this analysis is zinc oxide. It has been loaded with $5 \%$ and $10 \%$ of $\mathrm{Ga}$, which has an absorption edge of $10367 \mathrm{eV}$. The peak (100) was measured for the zinc oxide loaded with $10 \% \mathrm{Ga}$. In the case of $5 \% \mathrm{Ga}$, we measured peaks (100) and (101). With the information provided by the AXRD we can identify if Ga is being incorporated in the $\mathrm{ZnO}$ crystal structure. The analysis of 311 plane in the $\mathrm{ZnCo}_{2} \mathrm{O}_{4}$ spinel shows if Co is in tetrahedral or octahedral site.

\section{MATERIALS AND METHOD}

\section{Theoretical Model}

In a diffraction experiment, incident X-rays are directed toward a sample of interest, and are deflected by the atoms in the sample. The waves that are diffracted from different atoms can interfere with each other and consist of sharp interference maxima (peaks) with the same symmetry as in the distribution of atoms [4]. The atomic distance is an important fact in this analysis because it is directly related to the peaks in an X-ray diffraction patterns. For a given set of lattice planes with an inter-planar distance of $d$, the condition for a diffraction (peak) to occur can be simply written as which is known as the Bragg's law.

$$
n \lambda=2 d \sin \theta
$$


In this equation, $\lambda$ is the wavelength of the $\mathrm{X}$-ray, $\theta$ is the scattering angle, and $n$ is an integer representing the order of the diffraction peak (see Figure 4). It represents the scattering of radiation from two crystal planes of a crystal. These planes behave like half-reflecting mirrors. There is a difference of $2 d \sin \theta$ in the pathlength travelled by the two beams of radiation where $d$ is the perpendicular distance between the planes (the path difference is marked in green and the expanded construction alongside makes the geometry more clear). As the angle of reflection is changed so does the difference in pathlength travelled by the two beams. When the path difference is equal to an integer number of wavelengths the two beams will reinforce one another and when it is an integral number of half wavelengths the two waves will interfere destructively with one another. The intensity of the total reflected radiation will vary sinusoidally with $\theta$ [6][7]. Understanding and application of Bragg's Law can provide much useful information about various samples. It can identify the lattice parameter and phase of the material. Proper understanding of the structure factor can yield more information, particularly when combined with X-ray absorption for AXRD measurements.

$$
\begin{gathered}
F_{(h, k, l)}=\sum_{n=1}^{\text {atoms }} f_{n}(E) e^{(2 \pi i)\left(h x_{n}+k y_{n}+l z_{n}\right)} \\
f_{n}=f_{0}(Q)+f^{\prime}(E)+i f^{\prime \prime}(E)
\end{gathered}
$$

In this equation $f_{n}$ represents the atomic scattering factor, $x_{n}, y_{n}$, and $z_{n}$ are the fractional positions of the $\mathrm{n}^{\text {th }}$ atom, $f_{0}(Q)$ is the normal factor (E independent), $f^{\prime}(E)$ is the anomalous (E dependent), and $f^{\prime \prime}(E)$ is the absorption (E dependent). The $f_{n}$ varies near X-ray absorption edge, producing a total intensity change. One fact to be considered about $f_{n}$ is that it depends on 
oxidation state of the element [2]. Calculating the scattering factor let us know the intensity value since both are proportional.

$$
I_{h k l}=\left|F_{h k l}\right|^{2}
$$

In this equation, the intensity, $I$, is equal to the square of the absolute value of the scattering factor. For this reason, if the X-ray energy near the absorption edge is varied, then total intensity change.

\section{Data Analysis}

The software used to develop the analysis was Origin. Once the data was collected at SSRL beamline 2-1, we used Origin to fit peaks and obtain their integrated intensity (peaks area). As peaks are a combination of Gaussian and Lorentzian functions, we used the Pseudo Voigt function for fitting. Compared with Gaussian and Lorentzian functions, Pseudo Voigt gave us the lowest standard errors in the fittingFor each data set we selected some scans to make an average of the $\mathrm{w}_{\mathrm{G}}, \mathrm{w}_{\mathrm{L}}$, and $\mathrm{m}_{\mu}$. The reason we used the average values was to keep the peak shape constant for all X-ray energies.

When the peaks are fitted, we proceed to plot the obtained peak area values as a function of X-ray energies. If we see a feature at an energy corresponding to the absorption edge of a particular element, then that element is present at that particular crystallographic phase. In our case, we are looking for $\mathrm{Ga}$ in $\mathrm{ZnO}$, which has an absorption edge at $10367 \mathrm{eV}$, and for $\mathrm{Co}$ in $\mathrm{ZnCo}_{2} \mathrm{O}_{4}$ spinel, which has an absorption edge at around $7709 \mathrm{eV}$. The presence of the cation in 
the graph tells us about the material structure. We can determine if the atoms are in tetrahedral or octahedral site in the compound.

\section{RESULTS}

The average values obtained using Pseudo Voigt function were 0.5835 for $w_{G}, 1.2969$ for $w_{L}$, and -0.0196 in $m_{\mu}$. In the case of the Gaussian and Lorentzian fitting, the average values for each $w$ were 0.4976 and 0.6196 respectively. For the $\mathrm{ZnO}$ loaded with $\mathrm{Ga}$, the data obtained showed a smooth curve in the plot of area as a function of X-ray energies. It did not show any dip at the Ga-absorption edge (see Figures 5,6,7).

Figure 4 shows the fitting for the spinel $\mathrm{ZnCo}_{2} \mathrm{O}_{4}$ in 311 geometry. There is a big change around $7730 \mathrm{eV}$, which means that cobalt is present in the sample because its absorption edge is very close to that found in the fitting $(7709 \mathrm{eV})$. The 311 plane probes both tetrahedral and octahedral sites in the spinel crystal structure, so the "dip" seen near the Co absorption edge means that Co is found in one or both of these sites (see Figures 8,9).

\section{DISCUSSION AND CONCLUSIONS}

The absorption edge seen for $\mathrm{ZnCo}_{2} \mathrm{O}_{4}$ determined that $\mathrm{Co}$ is present in the sample in tetrahedral and octahedral structure. It means that we can find $\mathrm{Co}^{2+}$ and $\mathrm{Co}^{3+}$ in the plane 311 . With the obtained results is clear that AXRD is a used technique for the determination of the structure of a material since we were able to obtain the needed data to identify whether the Ga and Co were in tetrahedral or octahedral sites. In the case of the fitting, we can conclude that diffracted peak intensity decreases depending on elements present on diffracting planes. As the energy is varied 
through the absorption edge, there is a step function in diffracted intensity. In general, the structural information obtained via diffraction is combined with chemical information obtained via spectroscopy. At an absorption edge, there is a significant change in the atomic scattering factor, which in turn affects the structure factor. Thus by scanning energy while maintaining the Bragg condition for a particular atomic plane, it is possible to determine if certain elements are present based on changes in scattered intensity associated with each element's absorption edge.

\section{ACKNOWLEDGMENTS}

This work was supported by the U.S. Department of Energy, Office of Science, through the Summer Undergraduate Laboratory Internship Program (SULI) and Stanford Synchrotron Radiation Lightsource (SSRL) at SLAC National Accelerator Laboratory. I would especially like to thank my mentors Michael Toney, Sumohan Misra, and Joanna Bettinger for their guidance during the realization of my project. I would also like to thank Yezhou Shi and Rodrigo Noriega

for their help and explanations. Finally, I would also like to thank Stephen Rock and all the SULI staff at SLAC for give me the opportunity to work during this summer under their program.

\section{REFERENCES}

[1] Bettinger, J., Misra, S. Anomalous X-ray Diffraction (AXRD), California. 2010.

[2] Bettinger, J. Probing the Effects of Dopants, Defects, and Crystal Structure in Spinel Transparent Conducting Oxides for Photovoltaic Applicationsi, California.

[3] Granqvist, C. G., Transparent conductors as solar energy materials: A panoramic review, Department of Engineering Sciences, The Ångström Laboratory, Uppsala University, Uppsala, Sweeden. 2007.

[4] Pecharsky, V. K., Zavalij, P.Y., Fundamentals of Powder Diffraction and Structural Characterization of Materials, Page 146-152, Springer, New York. 2005. 
[5] Thomas, R.K., Simple Solids and their Surfaces [Online]. Available:

http://rkt.chem.ox.ac.uk/tutorials/surfaces/solids.html

[6] Introduction to X-ray Diffraction, Materials Research laboratory, University of California, Santa Barbara. 2010.

[7] Cullity, B.D., Stock, S.R., Elements of X-Ray Diffraction, Page 31-47, Prentice Hall, New Jersey. 2001. 


\section{FIGURES}

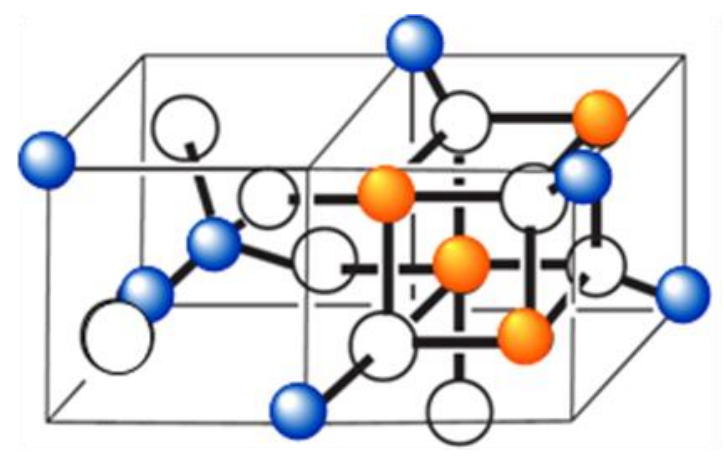

(a)

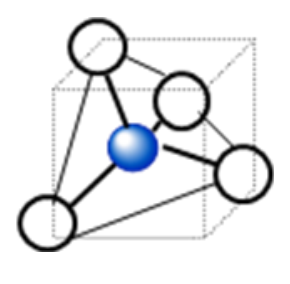

(b)

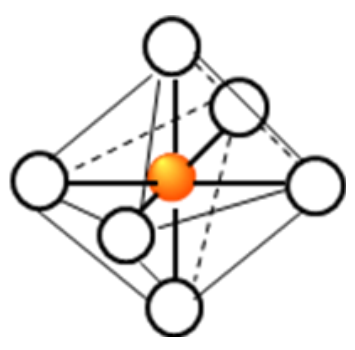

(c)

Figure 1: (a) Model of spinel $\mathrm{AB}_{2} \mathrm{O}_{4}$ structure (b) Model of tetrahedral sites in the spinel (c) Model of octahedral site in the spinel.

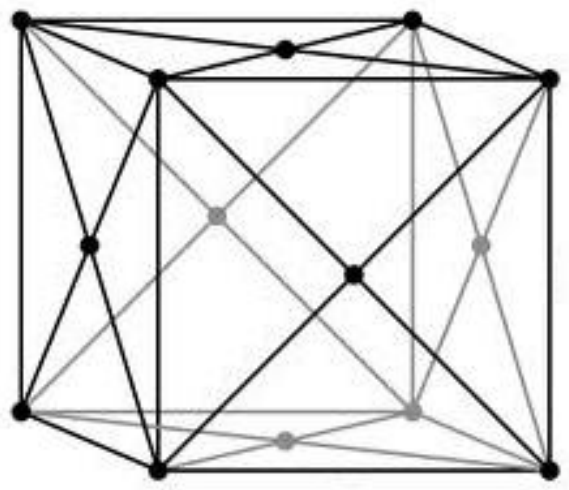

Figure 2: In a Face Centered Cubic (FCC) lattice the atoms are arranged at the corners and center of each cube face of the cell. 


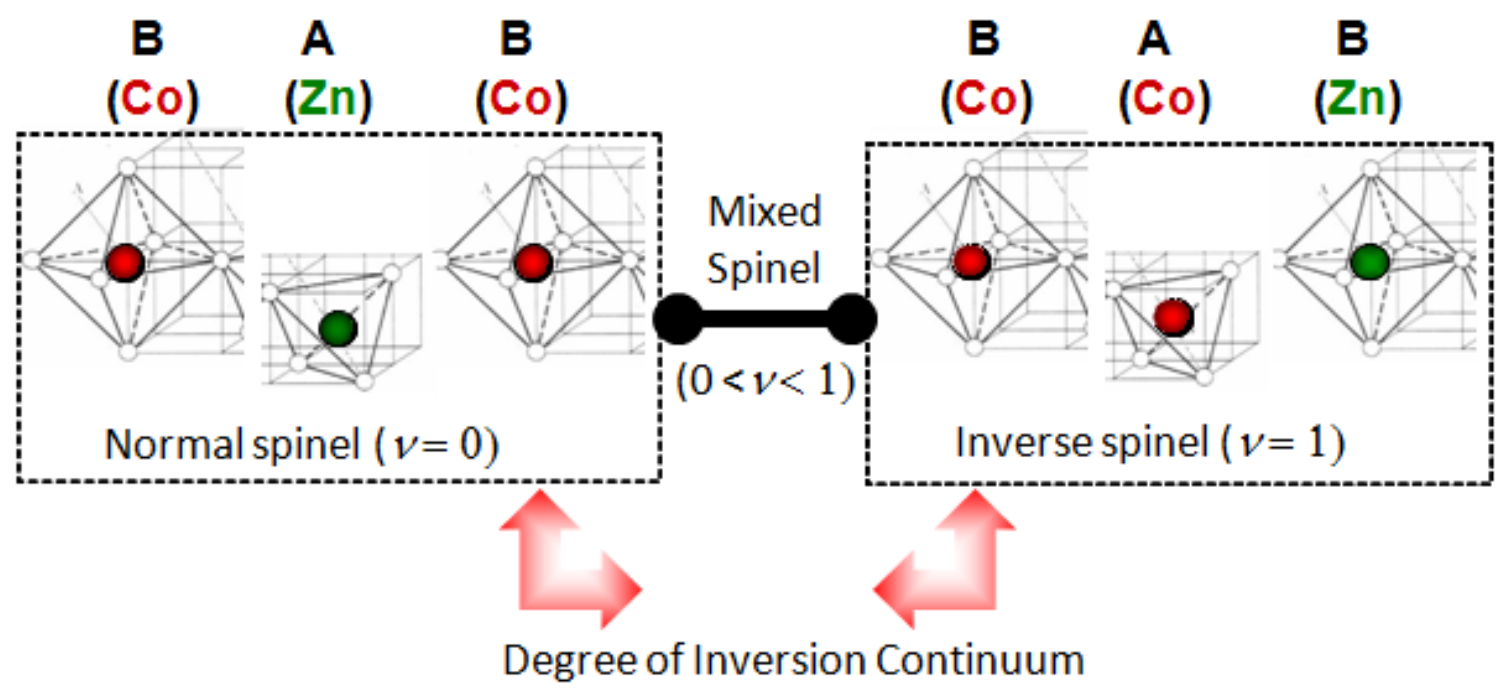

Figure 3: Representation of normal and inverse spinel of a $\mathrm{ZnCo}_{2} \mathrm{O}_{4}$. In the normal spinel $\mathrm{Zn}$ atom is in tetrahedral site and both Co atoms are in octahedral sites. For the inverse spinel, $\mathrm{Zn}$ is in octahedral site and atoms of Co are in tetrahedral and octahedral sites.

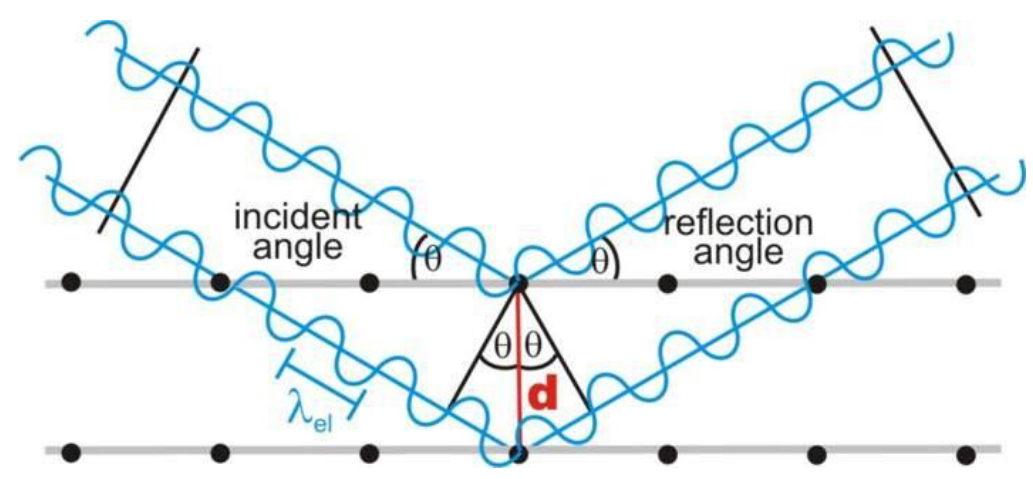

Figure 4: Model of Bragg's Law. Blue lines indicates the X-ray beamed and diffracted. $\theta$ is the angle formed by the diffraction, $d$ is the distance between the atoms plate 


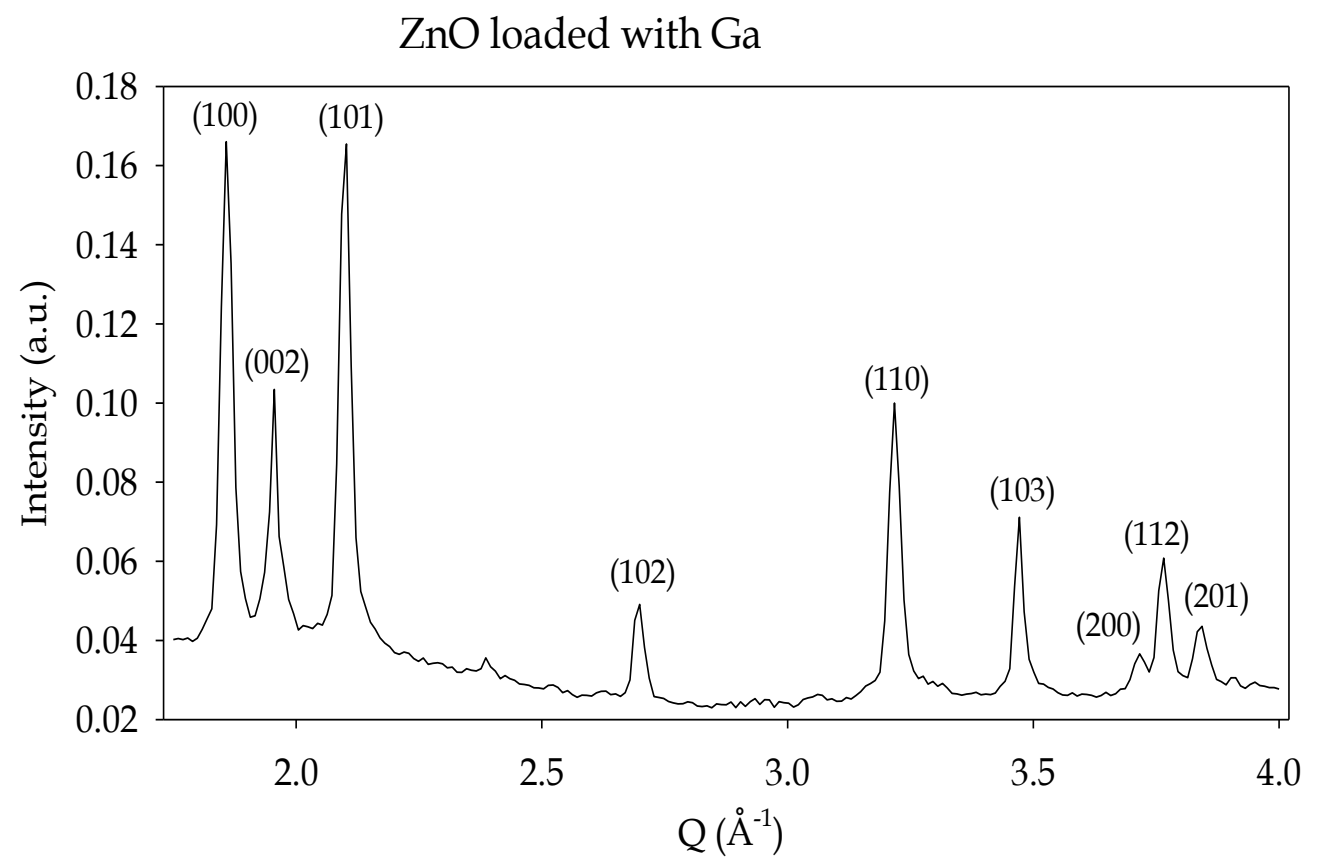

Figure 5: X-ray diffraction pattern for $\mathrm{ZnO}$. The peak (100) is analyzed using Origin software.

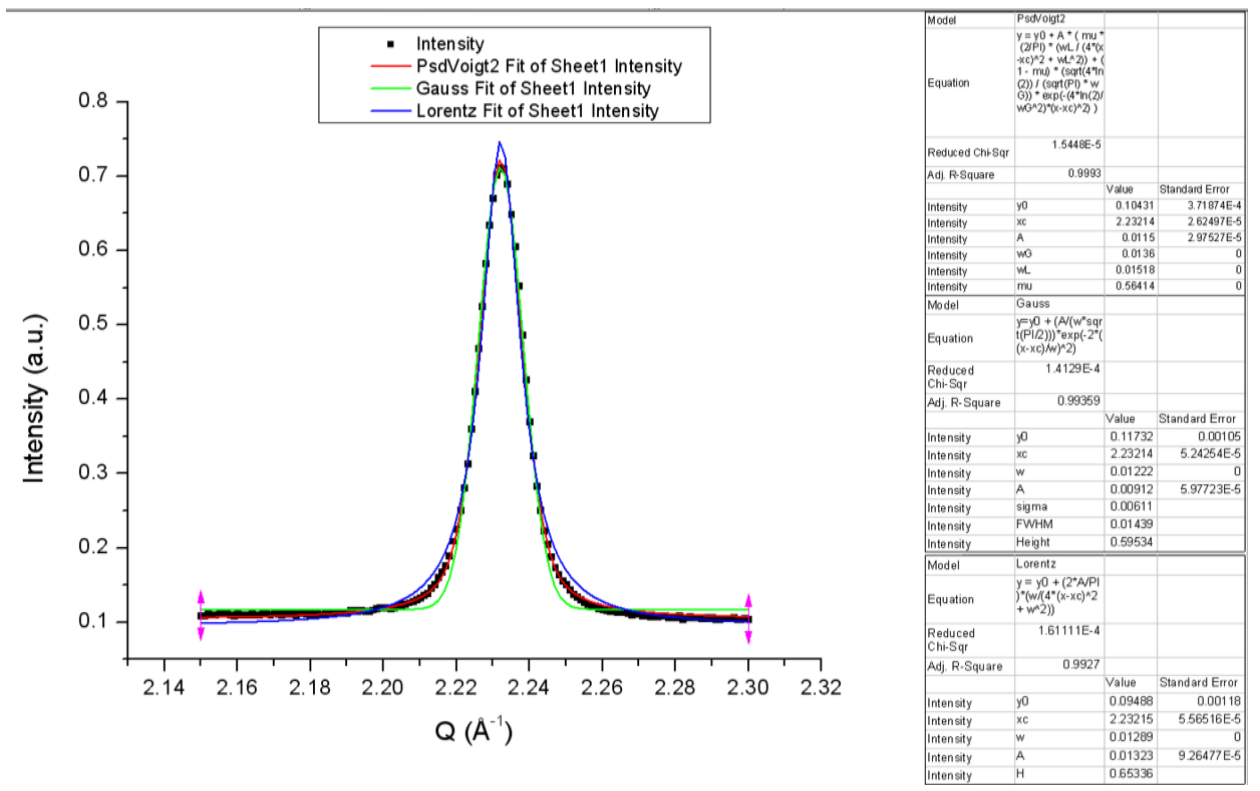

Figure 6: Fitting of peak (100) for scan 90 of $\mathrm{ZnO}$. Red line indicate the fiiting using Pseudo Voigt function, green line indicates the fitting using Gauss function, and blue line indicate the fitting using Lorentz function. 
- Pseudo Voigt

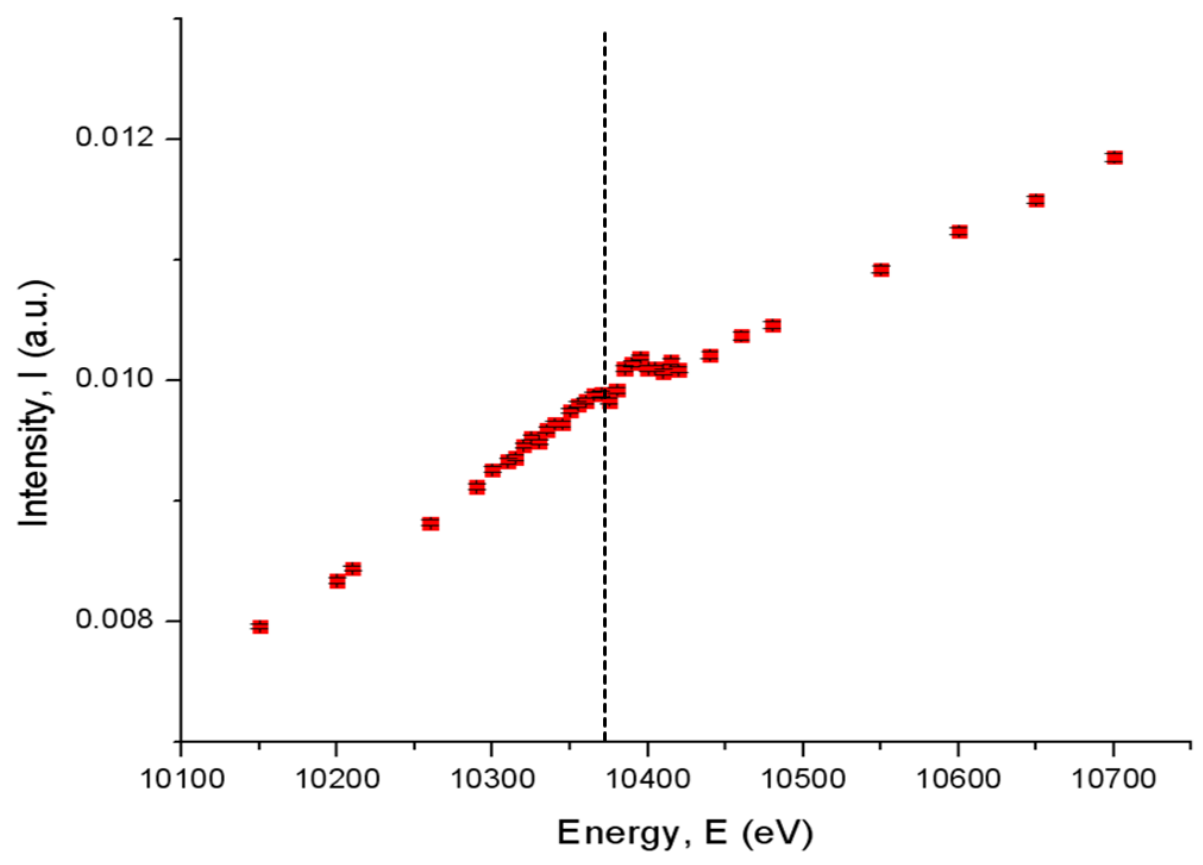

Figure 7: Graph of integrated intensity as a function of X-ray energy. Dashed line indicate Ga absorption edge at $10367 \mathrm{eV}$.

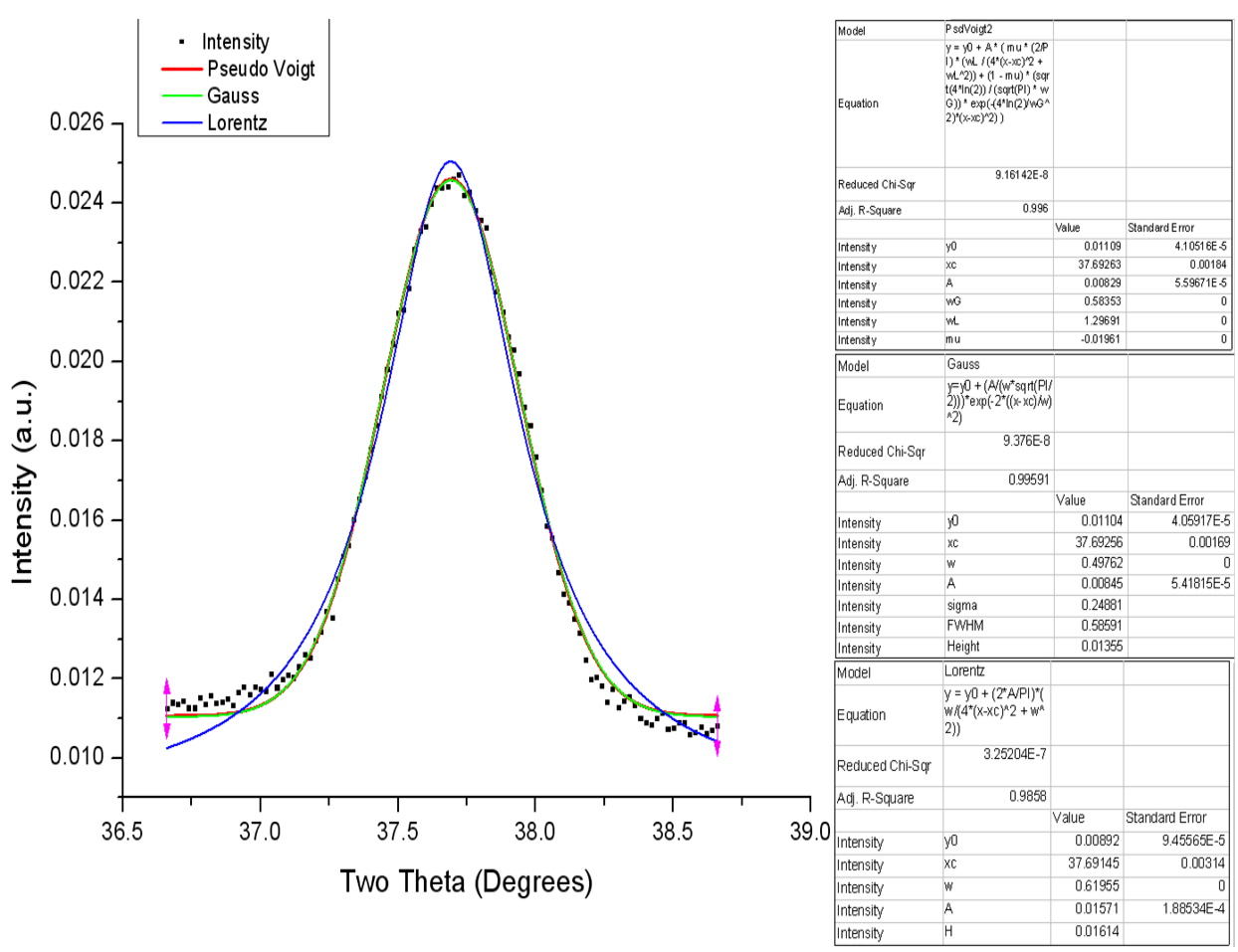

Figure 8: Fitting of peak (311) for scan 90 of $\mathrm{ZnCo}_{2} \mathrm{O}_{4}$ spinel. Red line indicate the fiiting using Pseudo Voigt function, green line indicates the fitting using Gauss function, and blue line indicate the fitting using Lorentz function. 
- Pseudo Voigt

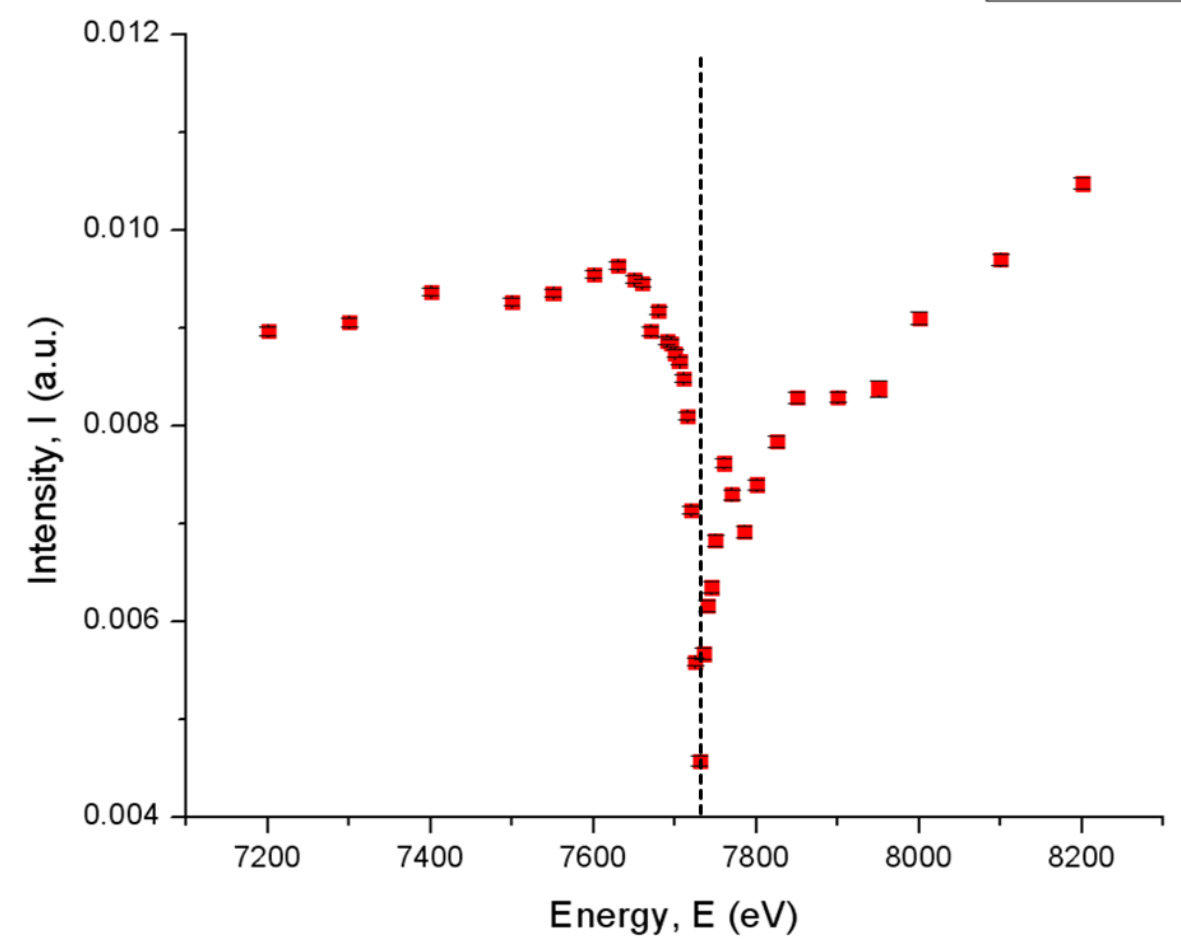

Figure 9: Graph of integrated intensity as a function of X-ray energy. Dashed line indicate Co absorption edge at $7709 \mathrm{eV}$. 